\title{
EIGENCONNECTIVITIES OF DYNAMIC FUNCTIONAL NETWORKS: CONSISTENCY ACROSS SUBJECTS
}

\author{
Nora Leonardi ${ }^{1,2}$, Dimitri Van De Ville ${ }^{1,2}$ \\ ${ }^{1}$ Institute of Bioengineering, École Polytechnique Fédérale de Lausanne (EPFL), Switzerland \\ ${ }^{2}$ Department of Radiology and Medical Informatics, University of Geneva, Switzerland
}

\begin{abstract}
Functional connectivity (FC) measured using fMRI has provided significant insights into brain function. However, increasing evidence points towards continuously fluctuating FC across the duration of a scan. Using unsupervised learning techniques, reproducible patterns of dynamic FC (dFC) have been revealed. In particular, based on principal component analysis, it has recently been proposed to represent $\mathrm{dFC}$ as a linear combination of multiple "eigenconnectivities". These group-level results were obtained by concatenating all subjects' timecourses of dFC. Here we investigate the consistency of these results by introducing a subject-level and group-level PCA and comparing the results with those obtained by concatenation.
\end{abstract}

Index Terms - functional MRI, dynamic functional connectivity, principal component analysis, canonical correlation analysis

\section{INTRODUCTION}

The use of functional magnetic resonance imaging (fMRI) to scan subjects during resting state has opened many opportunities for new advanced data processing techniques. The study of integration as an organizational principle of brain function heavily relies upon functional connectivity (FC), which is traditionally defined as Pearson correlation between the time series of two brain regions, computed over the whole scan (typically of several minutes). One recent avenue is to investigate non-stationarity of FC by introducing a slidingwindow variant and then explore FC fluctuations over time; the timedependent FC signal is referred to as dynamic FC (dFC).

Seminal work [1] has showed that fluctuations in dFC could be observed between major hubs of resting-state networks such as the default-mode network. The sliding-window approach should be used with care as to avoid spurious fluctuations introduced by the estimation method itself $[2,3,4]$, which can be avoided by proper high-pass filtering of the regional time series. Another difficulty for systematic whole-brain analysis is the number of connections that grows quadratically with the number of regions. Therefore, multivariate approaches such as clustering [5] or subspace decompositions [6, 7] have been proposed to reduce the dimensionality and analyze dynamics in a much lower dimensional space.

Here we investigate the consistency across subjects of "eigenconnectivities", which have been proposed previously [6] by our group to represent the group-level dFC data matrix by a limited number of "building blocks". We first briefly summarize the original approach, and the introduce the canonical correlation analysis (CCA)

This work was supported by the Swiss National Science Foundation (grant PP00P2-146318), the Center for Biomedical Imaging (CIBM) of Geneva and Lausanne Universities and the Leenards and Louis-Jeantet Foundations. and its generalization (gCCA) to study group-level eigenconnectivities that are driven by consistencies across subjects.

\section{DYNAMIC FUNCTIONAL CONNECTIVITY}

\subsection{Data description}

Subjects and MRI data were the same as in a previous study of our group [8], including 14 healthy controls and 22 minimally-disabled multiple sclerosis patients. The study was approved by the local institutional ethics committee and all subjects provided informed consent. Data were acquired on a Siemens 3T TrioTIM using a 32channel head coil. Functional imaging data were acquired in one session using gradient-echo planar imaging $(\mathrm{TE}=27 \mathrm{~ms}$, $\mathrm{TR}=1.1$ $\mathrm{s}$, flip angle $=90^{\circ}$, matrix $=64 \times 64,21$ transverse slices, voxel size $=3.75 \times 3.75 \times 5.63 \mathrm{~mm}^{3}, 450$ volumes). Subjects were instructed to lie still with their eyes closed and not to fall asleep (which was confirmed in a debriefing after the scan).

\subsection{Data preprocessing}

Anatomical and functional data were preprocessed using SPM8 and a combination of in-house MATLAB scripts and scripts from the DPARSFA toolbox [9]. The first ten functional volumes were discarded to allow for T1 equilibration effects. The other 440 functional volumes were spatially realigned to the mean image, detrended (linear and quadratic trends) and bandpass filtered $(0.02-0.15 \mathrm{~Hz})$. To further minimise spurious changes in connectivity related to motion, we "scrubbed" our data according to the procedure of Power et al. [10] and the criteria we specified in [6]. We excluded 1 HC subject and 7 RRMS patients with either maximal head motion above $3 \mathrm{~mm}$ or $2^{\circ}$.

The functional data were coregistered with each subject's anatomical data. The anatomical data were segmented (new segmentation algorithm of SPM8 [11]) and regionally parcellated using the automated anatomical labeling atlas, which divided the brain into 90 anatomical regions of interest [12]. The segmentation step provided a deformation field, which was used to warp the structural atlas into the subject's native space. We estimated regionally-averaged time series by averaging the fMRI signal over all voxels in each brain region. The time series from the bilateral globus pallidus were discarded due to ventral signal dropout in some subjects, leaving $N=88$ brain regions. We regressed out nuisance variables from the regional time series ( 6 head motion parameters, average cerebrospinal fluid from ventricular masks and white matter signal from white matter masks). The global signal was not regressed out. 


\subsection{Dynamic FC estimation}

We estimated $\mathrm{dFC}$ by calculating the pairwise Pearson correlation between the residuals of all $N$ brain regions' time series using a sliding-window technique yielding a $N \times N$ correlation matrix for each window; i.e., sliding-window correlation between the time series $x$ and $y$ was given by $\rho_{x y}(t)=\operatorname{corr}(x[t, t+\Delta t], y[t, t+\Delta t])$, where $\Delta t$ was the window length in TRs and the window was shifted by two TRs for each estimation. Correlations $\rho$ were Fisher r-to-z transformed $(z=\operatorname{atanh}(\rho))$ to make them approximately normally distributed.

We constructed a $\mathrm{dFC}$ data matrix $\mathbf{C}_{s}$ or each subject, where each column contained the unfolded upper-triangular part of the correlation matrix, resulting in a $\left(N^{2}-N\right) / 2 \times T_{s}$ matrix, where $T_{s}$ the number of windows for subject $s$. We normalized each subject's $\mathrm{dFC}$ matrix by removing the global mean and dividing by its standard deviation (mean and standard deviation over all time points and FC pairs). We used window length of 30 TRs covering 33 seconds.

\section{FC PATTERNS ESTIMATED BY CONCAT-PCA}

Following [6], we subtracted the row-wise means of $\mathbf{C}_{s}$ for each subject $s$ individually before we concatenated all subjects along the temporal dimension to establish a group-level $\mathrm{dFC}$ data matrix:

$$
\mathbf{X}=\left[\mathbf{C}_{1}-\overline{\mathbf{C}}_{1}, \mathbf{C}_{2}-\overline{\mathbf{C}}_{2}, \ldots, \mathbf{C}_{S}-\overline{\mathbf{C}}_{S}\right] .
$$

Centering allows to remove inter-subject variability of mean correlations, and thus to ensure that components are driven by fluctuations of correlation only. We then applied SVD to X, which finds the connection-wise and time-wise eigenvectors of the respective covariance matrices of $\mathbf{X}$; i.e., we have $\mathbf{X}=\mathbf{U} \Lambda \mathbf{V}^{T}$, where the columns of $\mathbf{U}$ are orthonormal eigenvectors termed "eigenconnectivities".

By convention, the eigenconnectivities are ranked according to decreasing explained variance (EV). Therefore, approximating the data using only the $K$ eigenconnectivities with the $K$ largest eigenvalues results in a $\mathrm{EV}$ of $\sum_{k=1}^{K} \lambda_{k}^{2} / \sum \lambda_{k}^{2}$.

A subject's dFC matrix can then be efficiently represented by projecting it onto the top- $K$ eigenconnectivities: $\mathbf{W}_{s}=\mathbf{U}_{\text {red }}^{T}\left(\mathbf{C}_{s}-\right.$ $\overline{\mathbf{C}}_{s}$ ), where $\mathbf{W}_{s}$ is a matrix of size $K \times T_{s}$ containing the weights of $K$ eigenconnectivities at all $T_{s}$ windows, and $\mathbf{U}_{\text {red }}$ contains only the first $K$ eigenconnectivities. These weights are comparable to the time-dependent profiles of "eigenimages" [13] and conceptually related to subject-dependent time series of independent component analysis (ICA) [14].

\section{FC PATTERNS ESTIMATED BY GENERALIZED CANONICAL CORRELATION ANALYSIS}

Here we propose to search for FC patterns that take commonalities across datasets, but not aspects specific to individual subjects, into account. A classical method to identify such common patterns is CCA [15], and gCCA for more than two datasets [16]. gCCA searches for a low-dimensional representation that takes statistical dependencies between datasets, as measured by their correlations, into account. If each dataset is whitened ${ }^{1}$ in a first step, gCCA,

\footnotetext{
${ }^{1}$ Whitening transforms a dataset $\mathbf{X}$ into a new dataset $\tilde{\mathbf{X}}$ whose covariance is the identity matrix, that is it is decorrelated and has unit variance. For row zero-mean data, the whitening transform can be found by solving the following eigenvalue decomposition: $\mathbf{X}^{T} \mathbf{X}=\mathbf{V} \boldsymbol{\Lambda} \mathbf{V}^{T}$. Whitened data $\tilde{\mathbf{X}}$ is then obtained as $\tilde{\mathbf{X}}=\mathbf{X V \Lambda} \boldsymbol{\Lambda}^{-1 / 2}$. Now $\tilde{\mathbf{X}}^{T} \tilde{\mathbf{X}}=$ $\mathbf{\Lambda}^{-\mathbf{1} / \mathbf{2}} \mathbf{V}^{T} \mathbf{X}^{T} \mathbf{X} \mathbf{V} \mathbf{\Lambda}^{-\mathbf{1} / \mathbf{2}}=\mathbf{I}$. $\tilde{\mathbf{X}}$ can also be directly obtained from a
}

however, simplifies to PCA. This property has for example been exploited in the analysis of gene expression [17] or as a preprocessing tool to enhance the reproducibility of ICA [18]. Specifically, [18] proposed a two-level model for multi-subject fMRI data, which is based on a subject-level PCA that reduces both the dimensionality of the data and subject-specific variability, that is followed by a group-level PCA (corresponding to a gCCA) capturing the remaining variability and identifying a common subspace.

We derive the equivalence of applying gCCA or the two steps of (i) whitening each subject's dFC and (ii) a group-level PCA. In the section thereafter we compare PCA and gCCA.

\section{1. gCCA and its relation to PCA}

We first introduce CCA, which is defined for two datasets [15]. For $S=2$ datasets $\mathbf{X}_{1}\left(C \times T_{1}\right)$ and $\mathbf{X}_{2}\left(C \times T_{2}\right)$, which in our case are two subject's centered dFC matrices, CCA maximizes the correlation between the projections $\mathbf{X}_{1} \mathbf{A}_{1}$ and $\mathbf{X}_{2} \mathbf{A}_{2}$ onto a $K$ dimensional common subspace, with $\mathbf{A}_{1}\left(T_{1} \times K\right)$, and $\mathbf{A}_{2}\left(T_{2} \times\right.$ $K)$. CCA can be solved using a generalized eigenvalue decomposition:

$$
\left[\begin{array}{cc}
0 & \mathbf{C}_{12} \\
\mathbf{C}_{21} & 0
\end{array}\right]\left[\begin{array}{l}
\mathbf{A}_{1} \\
\mathbf{A}_{2}
\end{array}\right]=\Lambda\left[\begin{array}{cc}
\mathbf{C}_{11} & 0 \\
0 & \mathbf{C}_{22}
\end{array}\right]\left[\begin{array}{l}
\mathbf{A}_{1} \\
\mathbf{A}_{2}
\end{array}\right]
$$

where $\mathbf{C}_{11}=\mathbf{X}_{\mathbf{1}}^{T} \mathbf{X}_{\mathbf{1}}$, and $\mathbf{C}_{22}=\mathbf{X}_{2}^{T} \mathbf{X}_{2}$ are the auto-covariance matrices, $\mathbf{C}_{12}=\mathbf{X}_{1}^{T} \mathbf{X}_{2}=\mathbf{C}_{21}^{T}$ is the cross-covariance matrix and $\Lambda$ contains the generalized eigenvalues on its diagonal.

For $S>2$, we use a generalization for which the solution is again given by a generalized eigenvalue decomposition [16, 18, 19]:

$$
\begin{array}{r}
{\left[\begin{array}{ccccc}
0 & \mathbf{C}_{12} & \mathbf{C}_{13} & \ldots & \mathbf{C}_{1 \mathbf{S}} \\
\mathbf{C}_{21} & 0 & \mathbf{C}_{23} & \ldots & \mathbf{C}_{2 \mathrm{~S}} \\
\vdots & & \ddots & & \vdots \\
\mathbf{C}_{\mathbf{S 1}} & \ldots & \ldots & \ldots & 0
\end{array}\right]\left[\begin{array}{c}
\mathbf{A}_{1} \\
\mathbf{A}_{2} \\
\vdots \\
\mathbf{A}_{\mathbf{S}}
\end{array}\right]} \\
=\Lambda\left[\begin{array}{cccc}
\mathbf{C}_{11} & 0 & \ldots & 0 \\
0 & \mathbf{C}_{22} & \ldots & 0 \\
\vdots & & \ddots & \vdots \\
0 & \ldots & \ldots & \mathbf{C}_{\mathrm{SS}}
\end{array}\right]\left[\begin{array}{c}
\mathbf{A}_{1} \\
\mathbf{A}_{2} \\
\vdots \\
\mathbf{A}_{\mathrm{S}}
\end{array}\right] .
\end{array}
$$

When all datasets are white, that is decorrelated and scaled to unit-variance $\left(\mathbf{X}_{s}^{T} \mathbf{X}_{s}=\mathbf{I}\right.$ for all $\left.s=1, \ldots, S\right)$, the CCA solution simplifies considerably:

$$
\left[\begin{array}{ccccc}
0 & \mathbf{C}_{12} & \mathbf{C}_{13} & \ldots & \mathbf{C}_{1 S} \\
\mathbf{C}_{21} & 0 & \mathbf{C}_{23} & \ldots & \mathbf{C}_{2 \mathrm{~S}} \\
\vdots & & \ddots & & \vdots \\
\mathbf{C}_{\mathbf{S} 1} & \ldots & \ldots & \ldots & 0
\end{array}\right] \mathbf{A}=\mathbf{\Lambda A}
$$

where $\mathbf{A}$ is the row-concatenated version of $\mathbf{A}_{s}$.

We now add IA to both sides of (2) because this allows us to express the cross-covariance matrix as the covariance matrix of the

SVD of $\mathbf{X}=\mathbf{U S V}^{T}$, where $\Lambda=\mathbf{S}^{2}$ and $\tilde{\mathbf{X}}=\mathbf{U}$. The dimensionality of the data can be reduced in the same step by retaining only the first $D$ eigenvectors of $\mathbf{U}$. 
concatenated, whitened datasets:

$$
\begin{aligned}
& {\left[\begin{array}{ccccc}
\mathbf{I} & \mathbf{C}_{12} & \mathbf{C}_{13} & \ldots & \mathbf{C}_{1 \mathbf{S}} \\
\mathbf{C}_{21} & \mathbf{I} & \mathbf{C}_{23} & \ldots & \mathbf{C}_{2 \mathbf{S}} \\
\vdots & & \ddots & & \vdots \\
\mathbf{C}_{\mathbf{S} 1} & \ldots & \ldots & \ldots & \mathbf{I}
\end{array}\right] \mathbf{A}} \\
& =\left[\begin{array}{c}
\mathbf{X}_{1}^{T} \\
\mathbf{X}_{\mathbf{2}}^{T} \\
\vdots \\
\mathbf{X}_{\mathbf{S}}^{T}
\end{array}\right]\left[\begin{array}{llll}
\mathbf{X}_{1} & \mathbf{X}_{2} & \ldots & \mathbf{X}_{\mathbf{S}}
\end{array}\right] \mathbf{A}=(\boldsymbol{\Lambda}+\mathbf{I}) \mathbf{A}
\end{aligned}
$$

The addition of the identity matrix leaves the eigenvectors unchanged and increases all eigenvalues by one. (3) describes a conventional PCA, completing the derivation.

In the original approach, we solved $\mathbf{X} \mathbf{X}^{T}=\mathbf{U} \boldsymbol{\Lambda} \mathbf{U}^{T}$, while (3) solves $\mathbf{X}^{T} \mathbf{X}=\mathbf{A}(\boldsymbol{\Lambda}+\mathbf{I}) \mathbf{A}^{T}$. $\mathbf{A}$ is therefore similar to what we previously called "weights", and we obtain the eigenconnectivities by projecting and normalizing: $\mathbf{U}=\mathbf{X A}(\boldsymbol{\Lambda}+\mathbf{I})^{-1 / 2}$. Or, simpler, by an SVD of the whitened data: $\mathbf{X}=\mathbf{U} \sqrt{(\boldsymbol{\Lambda}+\mathbf{I})} \mathbf{A}^{T}$. The eigenconnectivities obtained from concatenated subject eigenconnectivities (instead of dFC data) thus correspond to a subspace common to all subjects as obtained by gCCA.

\section{2. gCCA eigenconnectivities}

To compare PCA/SVD with gCCA, we applied SVD to each subject's dFC matrix and (arbitrarily) retained the first $19 \pm 2$ or $5 \pm 1$ eigenvectors, corresponding to $90 \%$ and $50 \%$ of EV, respectively (see Fig. 1b for an example spectrum of single subject's dFC). In Figs. $1 \mathrm{c}$ and e we show the first 10 eigenconnectivities obtained from whitened data. The eigenconnectivities strongly resemble those obtained without whitening (Figs. 1d and f), suggesting that the PCA patterns are not driven by subject-specific noise or dFC spikes, which would either not be retained after the subject-wise PCA or are unlikely to be shared by subjects. The eigenconnectivities are well preserved with as little as $50 \%$ of EV from the subject-level PCAs. With $90 \%$ of EV the eigenconnectivities are still largely similar, but less so than with $50 \%$ of EV, possibly because the whitening normalizes each subject's data to unit variance, which means that the $19^{\text {th }}$ principal component has a weight equal to the first one.

\section{CONCLUSION}

We introduced an approach to extract eigenconnectivities that are driven by inter-subject consistencies and provided first results. Future work should investigate how decompositions such as gCCA or agnostic CCA [20] would impact and potentially improve group differences (e.g., patients versus controls)

\section{REFERENCES}

[1] C. Chang and G. Glover, "Time-frequency dynamics of resting-state brain connectivity measured with fMRI," $\mathrm{Neu}$ roImage, vol. 50, no. 1, pp. 81-98, Mar. 2010.

[2] R. M. Hutchison, T. Womelsdorf, J. S. Gati, S. Everling, and R. S. Menon, "Resting-state networks show dynamic functional connectivity in awake humans and anesthetized macaques," Hum Brain Mapp, vol. 34, no. 9, pp. 2154-77, Mar 2013.

[3] D. A. Handwerker, V. Roopchansingh, J. Gonzalez-Castillo, and P. A. Bandettini, "Periodic changes in fMRI connectivity," NeuroImage, vol. 63, no. 3, pp. 1712-1719, Jul 2012.
[4] N. Leonardi and D. Van De Ville, "On spurious and real fluctuations of dynamic functional connectivity during rest," $\mathrm{Neu}$ roImage, vol. 104, pp. 430-436, January 2015.

[5] E. A. Allen, E. Damaraju, S. M. Plis, E. B. Erhardt, T. Eichele, and V. D. Calhoun, "Tracking whole-brain connectivity dynamics in the resting state," Cereb Cortex, vol. 24, no. 3, pp. 663-676, 2014.

[6] N. Leonardi, J. Richiardi, M. Gschwind, S. Simoni, J.-M. Annoni, M. Schluep, P. Vuilleumier, and D. Van De Ville, "Principal components of functional connectivity: A new approach to study dynamic brain connectivity during rest," NeuroImage, vol. 83, pp. 937-950, 2013.

[7] N. Leonardi, W. Shirer, M. Greicius, and D. Van De Ville, "Disentangling dynamic networks: Separated and joint expressions of functional connectivity patterns in time," Human Brain Mapping, vol. 35, no. 12, pp. 5984-5995, December 2014.

[8] J. Richiardi, M. Gschwind, S. Simioni, J.-M. Annoni, B. Greco, P. Hagmann, M. Schluep, P. Vuilleumier, and D. Van De Ville, "Classifying minimally disabled multiple sclerosis patients from resting state functional connectivity," NeuroImage, vol. 62, no. 3, pp. 2021-2033, September 2012.

[9] Y. Chao-Gan and Z. Yu-Feng, "DPARSF: A MATLAB toolbox for "pipeline" data analysis of resting-state fMRI.," Front Syst Neurosci, vol. 4, pp. 13, 2010.

[10] J. D. Power, K. A. Barnes, A. Z. Snyder, B. L. Schlaggar, and S. E. Petersen, "Spurious but systematic correlations in functional connectivity MRI networks arise from subject motion," Neuroimage, vol. 59, no. 3, pp. 2142-2154, Feb 2012.

[11] J. Ashburner and K. J. Friston, "Unified segmentation.," NeuroImage, vol. 26, no. 3, pp. 839-851, Jul 2005.

[12] N. Tzourio-Mazoyer, B. Landeau, D. Papathanassiou, F. Crivello, O. Etard, N. Delcroix, B. Mazoyer, and M. Joliot, "Automated anatomical labeling of activations in SPM using a macroscopic anatomical parcellation of the MNI MRI singlesubject brain," NeuroImage, vol. 15, no. 1, pp. 273-289, Jan. 2002.

[13] K. J. Friston, C. D. Frith, P. F. Liddle, and R. S. Frackowiak, "Functional connectivity: the principal-component analysis of large (pet) data sets.," J Cereb Blood Flow Metab, vol. 13, no. 1, pp. 5-14, Jan 1993.

[14] C. F. Beckmann, C. E. Mackay, N. Filippini, and S. M. Smith, "Group comparison of resting-state FMRI data using multisubject ICA and dual regression," in Proc. Organization for Human Brain Mapping (OHBM), San Francisco, CA, 2009, p. 1.

[15] H. Hotelling, "Relations between two sets of variates," Biometrika, vol. 28, pp. 321-377, 1936.

[16] J. Kettenring, "Canonical analysis of several sets of variables," Biometrika, vol. 58, no. 3, pp. 433-451, 1971.

[17] A. Tripathi, A. Klami, and S. Kaski, "Simple integrative preprocessing preserves what is shared in data sources," BMC Bioinformatics, vol. 9, no. 1, pp. 1-13, 2008.

[18] G. Varoquaux, S. Sadaghiani, P. Pinel, A. Kleinschmidt, J. B. Poline, and B. Thirion, "A group model for stable multi-subject ICA on fMRI datasets.," Neuroimage, vol. 51, no. 1, pp. 288299, May 2010.

[19] B. Afshin-Pour, G.-A. Hossein-Zadeh, S. C. Strother, and H. Soltanian-Zadeh, "Enhancing reproducibility of fMRI statistical maps using generalized canonical correlation analysis in NPAIRS framework.," NeuroImage, vol. 60, no. 4, pp. 1970 1981, May 2012.

[20] B. Afshin-Pour, C. Grady, and S. Strother, "Evaluation of spatio-temporal decomposition techniques for group analysis of fMRI resting state data sets.," NeuroImage, vol. 87, pp. 363-382, 2014. 

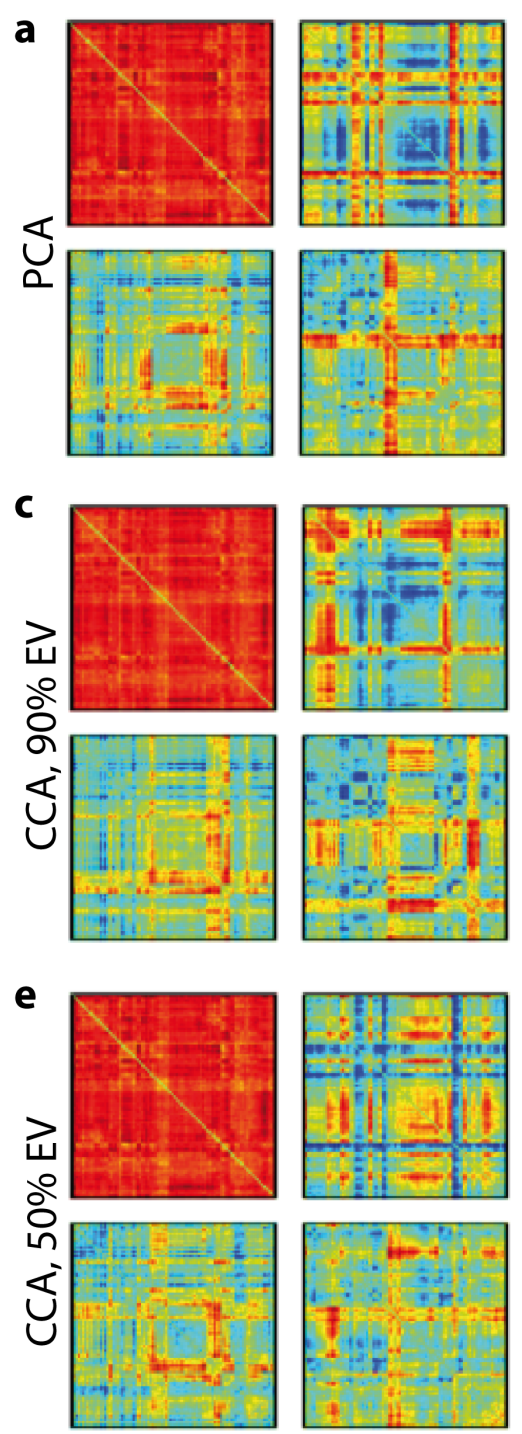
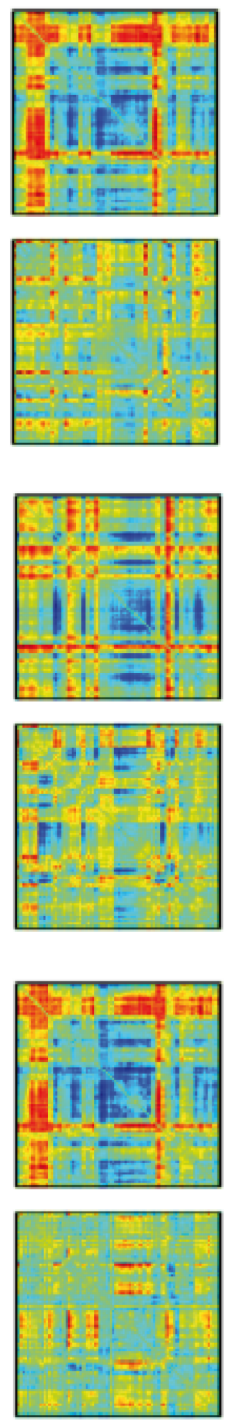
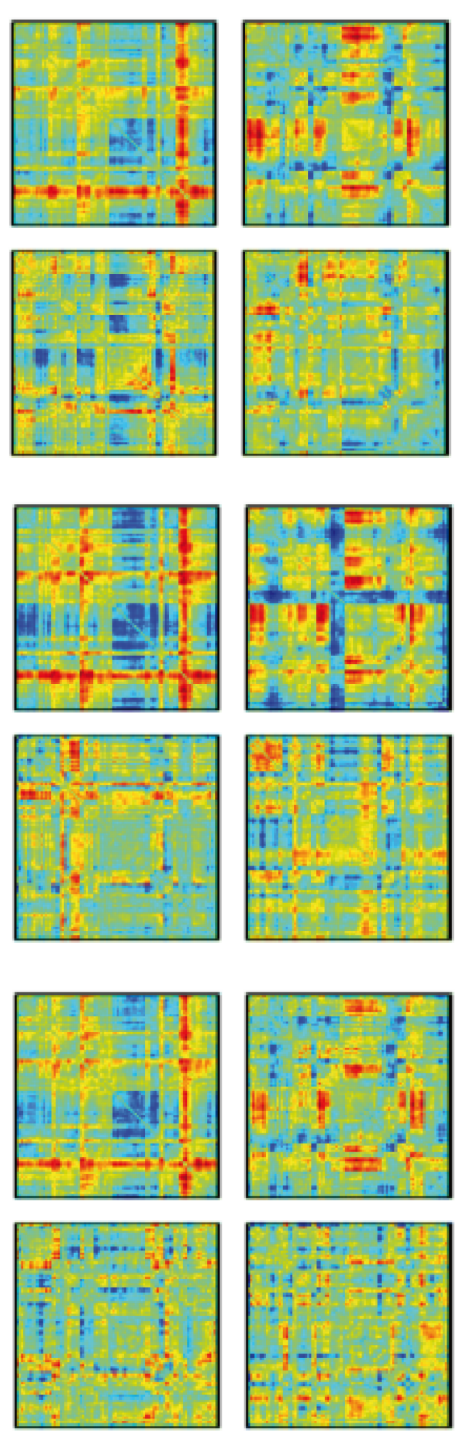
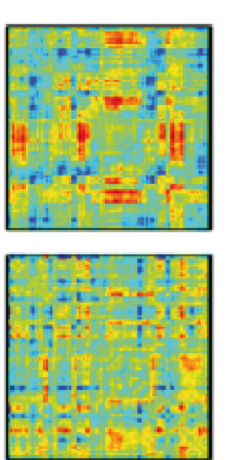

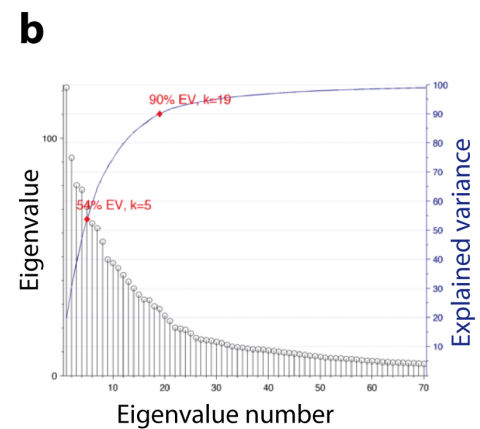

d Pearson correlation $r$
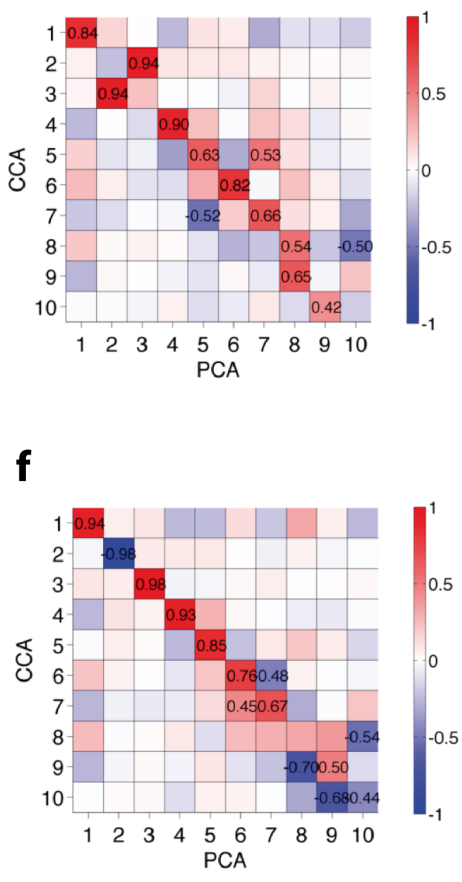

Fig. 1. (a) Eigenconnectivities obtained from concatenated dFC matrices. (b) Eigenvalues 1 to 70 out of 205 for a subject-wise PCA/SVD of $\mathrm{dFC}$, (c) eigenconnectivities obtained from a multi-subject PCA/SVD with $90 \%$ of EV for each subject's decomposition, (d) Pearson correlation $r$ between the patterns shown in (a) and (c), the values of correlations $r>0.4$ are indicated, (e) eigenconnectivities obtained from a multi-subject PCA/SVD with 50\% of EV for each subject's decomposition, (f) Pearson correlation between the patterns from (a) and (e). 